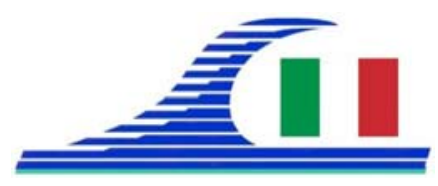

\author{
Conférence Méditerranéenne Côtière et Maritime \\ EDITION 3, FERRARA, ITALIA (2015) \\ Coastal and Maritime Mediterranean Conference \\ Disponible en ligne - http://www.paralia.fr - Available online
}

\title{
Le risque de tsunami dans quelques pays méditerranéens (France, Italie, Espagne et Turquie) : connaissance de l'aléa, perception et gestion du risque
}

\author{
Lydie GOELDNER-GIANELLA ${ }^{1,2}$, Delphine GRANCHER ${ }^{2}$, \\ Nuray KARANCI ${ }^{3}$, Nilay DOGULU ${ }^{3}$, Utku KANOGLU ${ }^{3}$, Stefano TINTI $^{4}$, \\ Filippo ZANIBONI ${ }^{4}$, Franck LAVIGNE ${ }^{1,2}$, Daniel BRUNSTEIN ${ }^{2}$
}

1. Université Paris 1 Panthéon-Sorbonne, France.

lydie.goeldner-gianella@univ-paris1.fr

2. CNRS, UMR 8591 Laboratoire de Géographie Physique, France.

3. Middle East Technical University, Turquie.

4. Dipartimento di Fisica e Astronomia, Università di Bologna, Italie.

\section{Résumé :}

Dans le cadre du programme de recherches européen ASTARTE (Assessment, STrategy And Risk reduction for Tsunamis in Europe), une enquête a été réalisée auprès de 1500 personnes fréquentant les plages et les rivages exposés à un risque de tsunami dans huit pays européens. L'article présente une première analyse des réponses de 1139 personnes - résidents et touristes - dans les sites exposés de quatre pays méditerranéens. Deux groupes ressortent - l'un composé de touristes interrogés en Espagne et en Turquie et d'Espagnols et de Turcs, l'autre de touristes questionnés en Italie et en France, de même que d'Italiens et de Français. Ils diffèrent par leur appréhension du risque de tsunami, leur degré de connaissance des tsunamis antérieurs et des caractéristiques de tsunamis potentiels, mais aussi leur croyance en un système d'alerte. Ces différences s'expliquent par des caractéristiques démographiques, géographiques ou culturelles. L'enquête fournit des éléments essentiels à la mise en place de systèmes d'information ciblés.

Mots-clés : Tsunami, Méditerranée, Perception, Gestion, Enquête.

\section{Introduction}

Les catastrophes de 2004 en Indonésie et 2011 au Japon ont témoigné de la vulnérabilité des sociétés et des usagers du littoral face à l'aléa "tsunami", qui se manifeste par une élévation anormale et rapide du niveau de la mer suivie d'une submersion des terres basses, littorales et insulaires. Or, le littoral européen s'avère lui aussi exposé aux tsunamis, alors qu'on y dénote a contrario une absence de culture de ce risque du fait de la relative rareté et de la faible ampleur des tsunamis qui s'y sont produits jusqu'à présent (DAWSON et al., 2004)., Néanmoins la Méditerranée est le théâtre d'environ un dixième des tsunamis recensés dans le monde depuis 1840 (TINTI et al., 2001). Plusieurs programmes de recherche ont porté sur ce risque, dont le projet MAREMOTI (MAREgraphie, observations de tsunaMis, mOdélisation et éTudes de vulnérabIIité 
Côtes méditerranéennes menacées :

Risques et défis dans le contexte du changement climatique

pour le nord-est Atlantique et la Méditerranée occidentale.), (2009-2012) de l'ANR RiskNat, centré sur l'observation, la modélisation et la vulnérabilité. Le projet européen ASTARTE (Assessment, STrategy And Risk reduction for Tsunamis in Europe) a adopté, pour sa part, une démarche d'amélioration de la connaissance et de la gestion de ce risque dans huit pays européens, en approchant le risque par l'association des géosciences et des sciences humaines. Quatre des sites méditerranéens du programme, tous identifiés comme vulnérables, sont présentés ici (fig. 1) : la commune touristique de Sant Jordi aux Baléares (E), la Côte d'Azur (F), la vieille ville de Syracuse en Sicile (I) et les rives d'Istanbul et de la baie de Gulluk (T). Ces sites sont exposés à des tsunamis d'origine sismique (ÁLVAREZ-GOMEZ et al., 2011 ; OKAL et al., 2004) ou volcanique ou causés par des glissements de terrain, sous-marins ou subaériens - tel celui de Nice en 1979 (SAHAL \& LEMAHIEU, 2011).

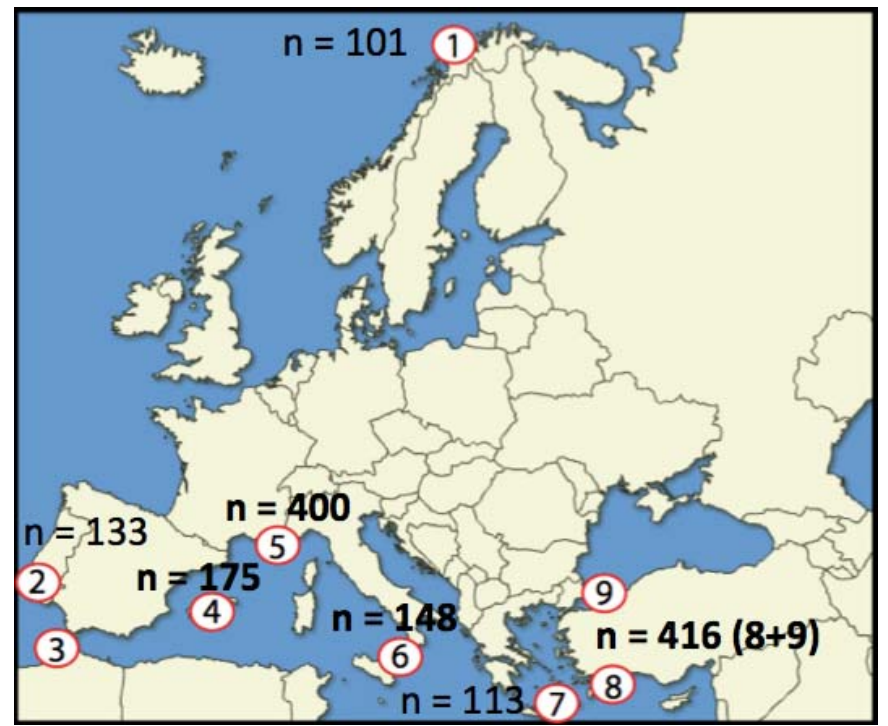

Figure 1. Sites du programme ASTARTE et nombre d'enquêtés : 1. Lyngen (N), 2. Sines (P), 3. Tanger (M), 4. Colonia St Jordi (E), 5. St-Laurent-du-Var, Nice, Villefran-chesur-mer (F), 6. Syracuse (I), 7. Héraklion (G), 8./9. Baie de Gulluk et Haydaparsa (T).

Les chercheurs et les autorités des pays méditerranéens s'efforcent de nos jours d'améliorer la gestion de ce risque, car il s'avère à forte gravité potentielle bien qu'il soit d'une faible probabilité. En France, depuis 2009, à la suite du rapport du sénateur COURTEAU (2007), le CEA, en partenariat avec le SHOM et le CNRS, pilote le nouveau centre d'alerte aux tsunamis (CENALT), chargé de surveiller l'Atlantique Nord-Est et la Méditerranée occidentale (donc les côtes françaises, espagnoles et italiennes, pour ce qui concerne notre étude) et de diffuser une alerte aux autorités françaises et aux autres centres d'alerte, notamment méditerranéens. Si des progrès ont été effectués dans l'alerte aux autorités régionales, il reste à faire parvenir l'information jusqu'aux autorités locales et surtout jusqu'aux résidents et usagers du littoral, puis à 
établir des plans locaux d'évacuation. Ce transfert d'information en cas d'alerte n'est en place dans aucun des sites d'étude, ce qui explique le recours à une enquête destinée à mieux évaluer la connaissance et les comportements spontanés des populations face à ce risque -aspects traités dans cet article-, de même que leur connaissance d'un éventuel système d'alerte et des comportements à adopter.

\section{Matériels et méthodes}

Cet article présente des données recueillies auprès de résidents et de vacanciers potentiellement exposés au risque de tsunami. S'inspirant de BIRD \& DOMINEYHOWES (2007), un questionnaire unique mais comprenant aussi des questions propres à chaque site a été déployé dans l'enquête ASTARTE, auprès de 1500 personnes, au printemps et à l'été 2014 et 2015 selon la disponibilité des enquêteurs. Le questionnaire, d'une durée de 15 à 30 minutes, se compose d'une cinquantaine de questions, majoritairement fermées. Elles portent sur la relation de la personne interrogée avec le site d'enquête, sa connaissance de l'aléa tsunami, son comportement en cas de tsunami, ses connaissances et opinion sur l'alerte donnée en cas de tsunami et son profil sociodémographique. Dans les quatre sites, les 1139 personnes interrogées (fig. 1) ont été abordées sur les plages et leurs abords. Les individus interrogés vivent ou travaillent dans le site d'enquête pour pratiquement la moitié d'entre eux (respectivement 34,4 \% de résidents et $13,2 \%$ de travailleurs locaux) mais font aussi du tourisme (52\%). Cet équilibre général est rompu en Espagne, où les touristes l'emportent (83\%). Les enquêtés d'origine locale prédominent dans les enquêtes menées en Italie, en Turquie et en France, suivis par des étrangers (Italie) ou des enquêtés d'origine nationale. L'enquête a ainsi été conduite auprès de 87 Espagnols, 106 Italiens, 343 Turcs et 403 Français. Ces enquêtés d'origine locale sont majoritairement des "résidents" en France et en Italie, alors qu'ils ont davantage le statut de "touristes" en Turquie, où ils ne résident plus mais se rendent en vacances. Ce qui distingue ces sites les uns des autres, c'est aussi la longue présence sur place des résidents interrogés en France et en Turquie (38\% des personnes interrogées y résident depuis plus de dix ans). Les personnes interrogées disposent d'un capital culturel légèrement plus élevé en Espagne et en France qu'en Italie et en Turquie. La répartition par sexe des personnes interrogées est plus équilibrée en France (49\% d'hommes) et en Italie, qu'en Espagne et surtout qu'en Turquie (61\% d'hommes). Si les enquêtés ont en moyenne plus d'une quarantaine d'années, les retraités sont particulièrement nombreux en France.

\section{Résultats}

En guise d'introduction et avant d'évoquer les tsunamis, il était demandé aux personnes interrogées de citer les risques pouvant potentiellement affecter la zone d'enquête question mettant en exergue la faible attention portée en Europe au risque de tsunami : celui-ci n'a été spontanément cité qu'en $4^{\text {ème }}$ position en France, $7^{\text {ème }}$ position dans la 
Côtes méditerranéennes menacées :

Risques et défis dans le contexte du changement climatique

baie de Gulluk $(\mathrm{T}), 9^{\text {ème }}$ position en Espagne et $11^{\text {ème }}$ position en Italie, derrière d'autres risques naturels ou anthropiques. Les enquêtés sont toutefois globalement capables de décrire un tsunami qu'ils associent à "la mer", une "grande" "vague", un "séisme", etc. Par ailleurs, concernant les signes annonciateurs d'un tsunami, les réponses générales de l'enquête dénotent aussi un certain niveau de connaissance : 43\% des personnes citent un tremblement de terre, $39 \%$ un retrait de la mer et 23\% un changement dans le comportement animal. Ces trois éléments ressortent systématiquement, mais pas forcément dans le même ordre, par site d'enquête ou nationalité : les personnes interrogées en Turquie -ou les Turcs- sont toutefois plus sensibles aux séismes et au comportement animal qu'au retrait de la mer, sans doute par connaissance de l'importance de ce risque dans ce pays. Pour autant, la grande majorité des enquêtés pense qu'aucun tsunami ne s'est produit par le passé dans la zone d'enquête - ou avoue son ignorance à Syracuse : cette méconnaissance est particulièrement élevée parmi les personnes interrogées en Turquie et en Espagne (entre 65 et 73\%). En revanche, les réponses données en France dénotent une certaine connaissance d'épisodes antérieurs de tsunami (28\%). C'est aussi les personnes interrogées en France (et en Italie) qui croient le plus en la survenue possible d'un tsunami dans la zone d'enquête (plus de $75 \%$ des réponses), alors qu'on y croit nettement moins lorsqu'on est interrogé en Turquie ou en Espagne (entre 37 et 42\%). Si l'on croise ces réponses avec la nationalité des individus, l'on obtient des résultats similaires : les Français et les Italiens sont apparemment bien plus pessimistes ou conscients qu'un risque de tsunami pourrait se produire dans la zone d'enquête, alors que les Espagnols et les Turcs sont beaucoup plus partagés et surtout plus nombreux à penser que ce ne sera pas le cas (plus de $40 \%$ contre $26 \%$ des personnes en moyenne dans l'enquête). On constate que les répondants sont influencés par les images qu'ils ont vues des tsunamis de 2004 et 2011, ce qui les amène à envisager de fortes hauteurs de vague pour les sites d'enquête considérés. Un tiers des enquêtés envisage une vague de $10 \mathrm{~m}$ et plus, un quart une vague de 5 à $10 \mathrm{~m}$ et $18 \%$ avouent leur ignorance. Par site, les résultats sont similaires, sauf en Turquie où les personnes interrogées ont été moins nombreuses à opter pour cette hauteur maximale. Par nationalité, les Turcs et les Espagnols imaginent majoritairement une vague plus faible (5-10 m ; et même 2 à $5 \mathrm{~m}$ en seconde position pour les Turcs). Comme mentionné supra, il semblerait que les Espagnols et les Turcs soient moins inquiets. Les enquêtés adoptent en général un comportement assez logique, lorsqu'ils ressentent un tremblement de terre ou voient une vague imposante à l'horizon (montrée sur photo). Dans le premier cas, 68\% quittent la plage, alors que 18\% y demeurent ; dans le second cas, $55 \%$ la quittent et moins de $7 \%$ y demeurent ; un tiers des personnes recherche même un site-refuge plus élevé. Les résultats qui s'écartent le plus de ces moyennes sont les suivants : les Français et surtout les personnes interrogées en France sont plus nombreux que la moyenne à rester sur les plages en cas de tremblement de terre, alors que les Turcs et les personnes interrogées en Turquie seraient plus nombreux à 
rechercher un site-refuge plus élevé, au contraire des Espagnols et des personnes interrogées à Majorque. Ce ne sont apparemment pas les sources de connaissance qui sont à l'origine de ces différences car trois d'entre elles sont indiquées par tous, dans le même ordre : la télévision (66\%), une couverture médiatique intense après un tsunami (41\%) et l'école (22\%), loin devant l'information publique (2\%) ! Seules des différences mineures affectent les réponses : les Turcs et les Italiens sont aussi informés par internet, les Français et les Espagnols par la radio. L'école joue un rôle moins légèrement marqué pour les Français. Enfin, alors qu'aucun système d'alerte aux individus n'existe dans les sites d'étude, les personnes interrogées en France et les Français sont plus nombreux que la moyenne à penser qu'il existe, au contraire des personnes interrogées en Turquie, des Turcs et des Espagnols.

\section{Conclusions}

Deux groupes se distinguent clairement au regard de cette première analyse. L'un, composé de personnes interrogées en Espagne et en Turquie et d'Espagnols et de Turcs, ne pense pas spontanément au risque de tsunami, méconnaît l'occurrence, dans le site d'enquête, de tsunamis antérieurs et ne croît pas en la survenue de futurs tsunamis, n'envisage que de faibles hauteurs de vagues et considère qu'un système d'alerte n'existe pas. La seule exception concerne la recherche d'un site-refuge plus élevé que les Turcs et les personnes interrogées en Turquie mettraient en œuvre. Certaines de ces réponses peuvent être liées, en Turquie, à la bonne connaissance du risque de tremblement de terre par la population ou à un capital culturel plus faible et une plus forte proportion d'hommes parmi les répondants. En Espagne, l'importance des touristes dans l'échantillon pourrait induire une sous-estimation du risque. Un second groupe, comprenant des individus interrogés en Italie et en France, des Italiens et des Français, pense plus spontanément à ce risque, connaît davantage l'occurrence, dans le site d'enquête, de tsunamis antérieurs et croît bien davantage en la survenue de futurs tsunamis, envisage de plus fortes hauteurs de vagues mais aussi l'existence d'un système d'alerte. Certaines caractéristiques de ce groupe, tels le nombre important de résidents locaux et de retraités ou la longue durée de résidence pourraient expliquer cette meilleure connaissance du risque. Une analyse statistique plus approfondie et la prise en compte d'autres critères - tels la religion, l'influence des media locaux ou le rôle des acteurs territoriaux - aideront à mieux interpréter ces différences.

Ces résultats d'enquête devraient inciter les autorités à informer bien davantage certaines populations locales ou la catégorie spécifique des touristes et ce, sur trois plans majeurs en chacun des sites : la possible survenue locale d'un tsunami, son amplitude potentielle et le fonctionnement des systèmes d'alerte - une fois ceux-ci mis en place localement. Ce sont donc des systèmes variés d'information qu'il faut établir par pays - voire par site - et par catégorie d'usager, de même que des systèmes d'alerte individuels visant bien l'ensemble des catégories (les résidents, mais aussi les touristes - 
Côtes méditerranéennes menacées :

Risques et défis dans le contexte du changement climatique

dont les perceptions du risque diffèrent souvent de celles des résidents (SEABRA et al., 2013). Au-delà d'une amélioration de l'information, l'enquête permettra aussi d'améliorer l'évacuation des sites - aspect non évoqué ici : les trajets que les usagers du littoral emprunteraient a priori pour fuir un tsunami (dessinés sur des cartes mentales) pourront en effet être comparés aux voies d'évacuation possibles qui seront également déterminées par les chercheurs du programme, afin, là encore, de définir la meilleure information à délivrer. Ces diverses mesures d'information sont essentielles pour faire gagner des minutes précieuses dans l'action, dans des secteurs très exposés.

\section{Remerciements}

ASTARTE bénéficie d'un soutien européen ( $n^{\circ} 603839$ 7th FP) et remercie l'équipe du METU et les étudiants de l'Université Paris 1 (C. Buchet, T. Bucourt, C. Charpentier, A. Cengil, S. Combe, M. Evans, J. Lenouvel, T. Lohrer, J. Lopes, T. Piot).

\section{Références bibliographiques}

ÁLVAREZ-GÓMEZ J.-A., ANIEL-QUIROGA I., GONZÁLEZ M., OTERO L. (2011). Tsunami hazard in the Western Mediterranean Spanish coast from seismic sources. Nat Hazards Earth Syst. Sci., Vol. 11, pp 227-240. http://dx.doi.org/10.5194/nhess-11-227-2011

BIRD D., DOMINEY-HOWES D. (2007). Testing the use of a questionnaire survey instrument to investigate public perceptions of tsunami hazard and risk in Sydney, Australia. Natural Hazards, Vol. 45(1), pp 99-122. http://dx.doi.org/10.1007/s11069-007-9172-8 COURTEAU R. (2007). Rapport sur l'évaluation et la prévention du risque de tsunami sur les côtes françaises en métropole et outre-mer, fait au nom de l'Office parlementaire d'évaluation des choix scientifiques et technologiques, n¹77 (2007-08), 168 p. URL : http://www.senat.fr/rap/r07-117/r07-1171.pdf

DAWSON A.-G., LOCKETT P., SHI S. (2004). Tsunami hazards in Europe. Environment International, Vol. 30(4), pp 577-585. http://dx.doi.org/10.1016/j.envint.2003.10.005 OKAL E. A., SYNOLAKIS C. E., USLU B., KALLIGERIS N., VOUKOUVALAS E. (2009). The 1956 earthquake and tsunami in Amorgos, Greece. Geophysical Journal International, Vol. 178(3), pp 1533-1554. http://dx.doi.org/10.1111/j.1365-246x.2009.04237.x SAHAL A., LEMAHIEU A. (2011). The 1979 Nice airport tsunami: mapping of the flood in Antibes. Natural Hazards, Vol. 56(3), pp 833-840. http://dx.doi.org/10.1007/s11069-010$\underline{9594-6}$

SEABRA C., DOLNICAR S., ABRANTES J.L., KASTENHOLZ E. (2013). Heterogeneity in risk and safety perceptions of international tourists. Tourism Management, Vol. 36, pp 502-510. http://dx.doi.org/10.1016/j.tourman.2012.09.008

TINTI S., MARAMAI A., GRAZIANI L. (2001). A new version of the European tsunami catalogue: updating and revision, Nat. Hazards Earth Syst. Sci., 1, pp 255-262. http://dx.doi.org/10.5194/nhess-1-255-2001 